\title{
Polymorphisms of genes encoding cytokines predict the risk of high-grade bladder cancer and outcomes of BCG immunotherapy
}

\author{
WOJCIECH KRAJEWSKI ${ }^{1}$, LIDIA KARABON ${ }^{1,2}$, ANNA PARTYKA ${ }^{2}$, ANNA TOMKIEWICZ2, \\ SŁAWOMIR POLETAJEW', ANDRZEJ TUKIENDORF ${ }^{4}$, ANNA KOEODZIEJ ${ }^{\prime}$, \\ ROMUALD ZDROJOWY \\ 'Department of Urology and Urological Oncology, Wroclaw Medical University, Wroclaw, Poland \\ ${ }^{2}$ Department of Experimental Therapy, Hirszfeld Institute of Immunology and Experimental Therapy, Polish Academy of Sciences, \\ Wroclaw, Poland \\ ${ }^{3}$ Second Department of Urology, Centre of Postgraduate Medical Education, Warsaw, Poland \\ ${ }^{4}$ Department of Social Medicine, Wroclaw Medical University, Wroclaw, Poland
}

\begin{abstract}
Introduction: The present study investigated the association of cytokines genes polymorphisms (IL-2, $I L-8$ and IL-18) and polymorphisms in genes encoding molecules related to the differentiation of Th17 subpopulation (IL-17 and IL-23R) with the risk of bladder cancer $(B C)$ and response to BCG immunotherapy.

Material and methods: Altogether, 175 BC patients treated with BCG due to high-grade non-muscle invasive tumors and 207 healthy individuals were genotyped for the following polymorphisms: IL-17A-197G >A (rs2275913); IL-17F+7488T>C (rs763780); IL-23Rc.309C>A (rs10889677); $I L-23 R c .1142 G>A(r s 11209026) ; I L-2-330 T>G(r s 2069762), I L-8-251 A>T(r s 4073)$, and IL-18-137G $>C$ (rs187238) using the TaqMan SNP genotyping assays.

Results: The IL-23Rc.-309C>A[A] allele was associated with the risk of BC (OR: 1.42, $p=0.03)$. Moreover, heterozygocities for IL-17A-197G $>A[G A]$ and IL-18-137G $>C[G C]$ increased the risk of BC, as compared to both homozygotes (OR: $1.67, p=0.01$ and OR: $1.84, p=0.008$, respectively). The IL-18$137 G>C[G C]$ heterozygous patients had the highest risk of tumor recurrence and progression, and the worst recurrence-free and progression-free survival. Homozygous IL-17A-197G $>$ A[GG] patients presented the best recurrence-free survival, while IL-17A-197G>A[AA] patients had 1.8-fold higher risk of recurrence.

Conclusions: The present study highlighted the importance of $I L-17, I L-18$, and IL-23R gene polymorphisms for BC susceptibility and BCG immunotherapy outcomes. It may help to identify appropriate candidates for early radical treatment.
\end{abstract}

Key words: gene polymorphisms, non-muscle-invasive bladder cancer, bacillus Calmette-Guerin, recurrence, progression.

(Centr Eur J Immunol 2020; 45 (1): 37-47)

\section{Introduction}

The bladder cancer (BC) is the fourth most prevalent malignancy and the second most frequently diagnosed urologic cancer in men. In 2016, 76,960 new cases of the disease and 16,390 cancer-specific deaths were observed in the United States only [1].

Bladder cancers can be divided into two main subgroups: non-muscle invasive BCs (NMIBC) and muscle invasive BCs (MIBC). NMIBCs is a BC's subclass that comprises tumors limited to mucosa and/or invading sub- mucosa (stages Ta, T1, and carcinoma in situ-Cis), while muscle invasive BC (stage T2 and more) infiltrates or passes the bladder muscular layer (lamina muscularis propria). At the time of diagnosis, a subgroup of NMIBC represents majority of BC cases [2].

The BC etiology is multifactorial. The best documented risk factor for $\mathrm{BC}$ development is both active and passive smoking; however, other factors such as exposure to aromatic compounds, use of cyclophosphamide and phenacetin, and previous radiotherapy have also been demon-

Correspondence: Wojciech Krajewski, Department of Urology and Oncological Urology, Wroclaw Medical University, 213 Borowska St., 50-556 Wroclaw, Poland, e-mail: wk@ softstar.pl Submitted: 25.12.2018; Accepted: 23.04.2019 
strated to play an important role in BC development [2]. Also, similarly to other malignancies, substantial influence of chronic inflammation and immunological processes in the bladder wall on its cancerous transformation have been proved [3, 4].

Standard of care in NMIBCs consists of transurethral resection of bladder tumor (TURB) with additional intravesical therapy, which for intermediate and high-risk NMIBCs is bacillus Calmette-Guerin (BCG) immunotherapy. It is documented that BCG action is based on immunological anti-tumor response. Components of the BCG instillation such as attenuated bacteria, dead bacilli, and subcellular debris are characterized by powerful immunological properties. After BCG instillation, an increased immunological activity in the bladder leads to the activation and migration of cytotoxic lymphocytes, macrophages, and NK cells, which finally results in tumor eradication [5]. However, despite the fact that BCG in NMIBC treatment was introduced more than 40 years ago, the precise therapeutic mechanism remains unclear [6].

It is widely known that cytokines are key mediators of the immune response. Among others, they mediate and regulate immunity, inflammation, and hematopoiesis. Therefore, any misbalance in ratio of Th1/Th2 types of immune response can lead to auto-immune, inflammatory, and malignant diseases. What is more, single nucleotide polymorphisms (SNPs) in genes that encode cytokines have been shown to alter their expression and modify their activity.

The role of variations in genes that encode cytokines may be analyzed in two aspects: as risk factor for BC development and as predictive factor of the antitumor therapy outcome. Therefore, the role for functional SNPs in key genes of the immune system and inflammatory response has been proposed. As proven before, the presence of polymorphisms in those genes may determine inter-individual differences in the susceptibility to cancer as well as might define response to treatment $[7,8]$.

The available literature data about role of cytokine genes polymorphisms with susceptibility to $\mathrm{BC}$ is very limited [9]. This is even more applicable in relation to response to BCG therapy in $\mathrm{BC}$ patients.

The aim of the study was to investigate the relationship between polymorphisms in genes encoding interleukines (IL-2, IL-8, and IL-18) as well as in genes related to the differentiation of $\mathrm{T}$ helper 17 cells subpopulation (IL-17 and IL-23R) and the risk of BC. Additionally, potential predictive capabilities of genetic biomarkers for BCG immunotherapy outcome were analyzed.

\section{Material and methods}

\section{Patients}

An institutional ethics committee approved the study and all patients gave written informed consent. Altogether,
175 histologically confirmed high-grade (HG) bladder cancer patients were recruited between March and September 2015 from one university center.

All patients included in this study were treated with BCG immunotherapy due to T1HG and Cis tumors, and terminated the therapy at least 36 months before the study onset [10]. Every subject participating in the study was genetically unrelated and Polish ethnicity. Before BCG immunotherapy onset, all of the patients underwent a TURB. A single, immediate post-operative intravesical instillation of chemotherapy and a re-staging resection (reTURB) was performed in patients according to the European Association of Urology clinical guidelines and urologists' latitude basing on pathologic and/or intraoperative outcomes. BCG immunotherapy was introduced at least 14 days after the last invasive bladder procedure. Whole BCG immunotherapy schedule consisted of 27 instillations divided into induction and maintenance courses. Inducting course was composed of six weekly given instillations. Maintenance courses were composed of three weekly given instillations administered after 3, 6, 12, 18, 24, 30, and 36 months [11]. Patients with at least 7 instillations (complete induction course and any maintenance) were included in the analysis. Cystoscopy was performed every 3 months during first two years following TURB and then every 6 months. After five years, cystoscopy was performed annually. Urine cytology was performed every 3 months if the primary tumor was poorly differentiated, or in case of concomitant Cis or mucosal dysplasia. In case of Cis, mapping biopsy was performed routinely after the first maintenance course. A recurrence was defined as a re-occurrence of tumor of any stage and grade confirmed by TURB and histologic or cytological assessment. A progression was defined as an increase to T2 or higher stage. Clinical data on tumor size, grade, stage, presence of carcinoma in situ concomitant, tumor focality, intravesical therapy as well as follow-up details containing dates of recurrence, progression and toxicity events, systemic chemotherapy, radical cystectomy, and pathologic results were summarized from patients' charts. The investigating urologist explained to each individual included in the project the purpose of the study, protection of participants' confidentiality and freedom to drop out at any time.

Two hundred and seven controls [155M/52F] without a history of cancer were recruited mainly from volunteer blood donors. Every subject participating in the study was genetically unrelated, with Caucasian ethnicity originating from the same geographical region of Poland. Patients baseline characteristics is presented in Table 1.

\section{Molecular analysis}

\section{Selection of single nucleotide polymorphisms}

For our study, we have selected SNPs located in promoter, intron, exon, or 3'UTR regions of genes, which 
were described in literature as functional [12-17] or on the basis of SNPinfo software [18]. Moreover, the minor allele frequency of selected polymorphisms for Caucasian population was not lower than $9 \%$.

\section{DNA isolation and genotyping}

Each individuals' DNA was isolated from white blood cells pellet from $4 \mathrm{ml}$ of venous blood by using QIAamp DNA blood mini kit (Qiagen, Germany) according to the manufacturer's protocol.

The following SNPs: IL-17A -197G>A (rs2275913); $\mathrm{IL}-17 \mathrm{~F}+7488 \mathrm{~T}>\mathrm{C} \quad(\mathrm{rs} 763780) ; \mathrm{IL}-23 \mathrm{R}$ c.309C >A (rs10889677); IL-23R c.1142G >A (rs11209026); IL-2 -330T>G (rs2069762), IL-8 -251A >T (rs4073), and IL-18 $-137 \mathrm{G}>\mathrm{C}$ (rs 187238) were genotyped in 175 patients and in 207 controls using TaqMan ${ }^{\circledR}$ SNP genotyping assays (C_15879983_10, C__2234166_10, C_11283764_10, C__1272298_10, C_15859930_10, C__11748116_10, and C__2408543_10, respectively). Genotyping was performed on ViiA 7 (AppliedBiosystems) equipment. Additionally, the results of $I L-18-137 \mathrm{G}>\mathrm{C}$ (rs187238) were verified using LightSNiP for rs187238 (TIB MolBiol) on the LightCycler 480 (Roche) instrument.

\section{Statistical analysis}

The study groups were compared using Pearson chisquare test for sex, gender, and primary diagnosis. For genotypes, the Hardy-Weinberg equilibrium (HWE) tests were analyzed. The associations between disease occurrence and genotypes and gene-gene interactions were assessed with Pearson chi-square test by calculating OR (odds ratio) and 95\% CI (confidence interval). The haplotype frequencies were determined using the SHEsis program [17]. Haplotypes with frequencies $<0.03$ were not considered. Recurrence-free survival (RFS) and progression-free survival (PFS) were estimated with the logrank method. Cox regression analysis was also performed. Survivors without any expected event of interest were censored at the date of the last follow-up. Analyses were conducted with SPSS software (version 22.0, SPSS Inc., Chicago, III., USA). Differences were considered statistically significant if $p<0.05$.

\section{Results}

Altogether, 382 individuals (175 BC patients and 207 controls) were analyzed in the study.

\section{Response to the BCG treatment}

Among 175 BC patients treated with BCG immunotherapy, 44 patients completed the whole BCG regimen (27 instillations). However, the average number of BCG instillations given was 18.7. The patients were followed from 22 to 235 months (median, 53). A recurrence was
Table 1. Patients' baseline characteristics (cases)

\begin{tabular}{lc}
\hline Parameter & $\begin{array}{c}\text { NMIBC patients } \\
\boldsymbol{n}=\mathbf{1 7 5}(\mathbf{\%})\end{array}$ \\
\hline Gender (M/F) & $134 / 41(76.6 / 23.4)$ \\
\hline Age (mean; SD) & $66 ; 9.5$ \\
\hline Primary diagnosis (T1HG/Cis) & $133 / 42(76 / 24)$ \\
\hline Concomitant Cis & $39(29.3)$ \\
\hline Number of tumors (solitary/multiple) & $68 / 65(51.1 / 48.9)$ \\
\hline Tumor size (<3 cm/3 cm) & $74 / 59(55.6 / 44.4)$ \\
\hline Newly diagnosed/recurrent & $111 / 64(63.4 / 36.6)$ \\
\hline Observation time (median; SD) & $53 ; 37.1$ \\
\hline Recurrence & $79(45.1)$ \\
\hline Progression & $49(28)$ \\
\hline Cystectomy & $45(25.7)$ \\
\hline NMIBC - non-muscle invasive bladder cancer, $M-$ male, F-female, \\
SD - standard deviation, Cis - carcinoma in situ
\end{tabular}

observed in 79 cases (45.1\%). Twenty-eight patients (16\%) experienced a recurrence more than once. Progression of the cancer was observed in 49 patients $(28 \%)$. Eventually, $45(25.7 \%)$ patients underwent radical cystectomy.

\section{The analysis of Hardy-Weinberg equilibrium}

The HWE was calculated for every polymorphism either for the patients and for the controls (Table 2). Slight deviation from the equilibrium was observed for IL-17A $-197 \mathrm{G}>\mathrm{A}$ polymorphism in BC population. This might indicate an association of this polymorphisms with the disease, since, what was proven before, cases do not need to be in HWE. Additionally, according to Lee et.al., deviations from $\mathrm{HWE}$ in disease affected populations are a sufficient proof to determine the relationship with the disease [19]. The deviation from HWE was also observed for IL-18 -137G>C polymorphism in control population. Therefore, the results were verified by the technique based on the different principle, i.e. LightSNiP method. Both results were consistent in $>99 \%$ (1/175 mismatch in BC and 1/207 in controls) and the deviation from HWE was still observed in the controls. Because of the fact that other polymorphisms were in HWE equilibrium for both controls and patients, we concluded that the control population was properly established.

\section{Association of the polymorphisms with the disease}

The genotype distributions of the SNPs in the patients and controls are shown in Table 2. There were no statistical differences in alleles distribution in the primary diagnosis subgroups (T1HG or Cis). The alleles distribution IL23R c.309 C/A polymorphism was significantly different in both study groups $\left(\chi^{2}=4.57, p=0.03\right)$, and the genotype distribution presented borderline difference between both 
Wojciech Krajewski et al.

Table 2. Genotype and allele frequencies among the cases and controls, and the association with risk of bladder cancer

\begin{tabular}{|c|c|c|c|c|}
\hline Genotype & $\begin{array}{c}\text { Patients } \\
n(\%)\end{array}$ & $\begin{array}{c}\text { Controls } \\
n(\%)\end{array}$ & OR $(95 \% \mathrm{CI})$ & $p$-value \\
\hline \multicolumn{5}{|c|}{ IL-18 -137G>C (rs187238) } \\
\hline GG & $80(45.7)$ & $117(56.5)$ & Ref. & \\
\hline GC & $83(47.4)$ & $68(32.9)$ & $1.84(1.22-2.79)$ & 0.008 \\
\hline $\mathrm{CC}$ & $12(6.9)$ & $22(10.6)$ & $0.80(0.37-1.70)$ & 0.56 \\
\hline $\mathrm{GC}+\mathrm{CC}$ & $95(54.3)$ & $90(43.5)$ & $1.54(1.03-2.31)$ & 0.04 \\
\hline HWE & $p=0.12$ & $p=0.02$ & & \\
\hline G & $243(69.4)$ & $302(72.9)$ & Ref. & \\
\hline $\mathrm{C}$ & $107(30.6)$ & $112(27.1)$ & $0.84(0.62-1.15)$ & 0.28 \\
\hline \multicolumn{5}{|c|}{ IL-17A -197G >A (rs2275913) } \\
\hline GG & $62(35.4)$ & $87(42.0)$ & Ref. & \\
\hline GA & $97(55.4)$ & $89(43.0)$ & $1.53(0.99-2.36)$ & 0.06 \\
\hline AA & $16(9.1)$ & $31(15.0)$ & $0.72(0.36-1.44)$ & 0.36 \\
\hline $\mathrm{GA}+\mathrm{AA}$ & $113(64.6)$ & $120(58.0)$ & $1.32(0.87-2.00)$ & 0.19 \\
\hline HWE & $p=0.01$ & $p=0.30$ & & \\
\hline G & $221(63.1)$ & $263(63.5)$ & Ref. & \\
\hline A & $129(36.9)$ & $151(36.5)$ & $1.02(0.76-1.37)$ & 0.91 \\
\hline \multicolumn{5}{|c|}{ IL-17F +7488T>C (rs763780) } \\
\hline TT & $162(92.6)$ & $187(90.3)$ & Ref. & \\
\hline $\mathrm{TC}$ & $13(7.4)$ & $20(9.7)$ & $0.75(0.36-1.56)$ & 0.44 \\
\hline $\mathrm{CC}$ & 0 & 0 & & \\
\hline $\mathrm{TC}+\mathrm{CC}$ & $13(7.4)$ & $20(9.7)$ & $0.75(0.36-1.56)$ & 0.44 \\
\hline HWE & $p=0.61$ & $p=0.47$ & & \\
\hline $\mathrm{T}$ & $337(96.3)$ & $394(95.2)$ & Ref. & \\
\hline $\mathrm{C}$ & $13(3.7)$ & $20(4.8)$ & $0.76(0.37-1.55)$ & 0.45 \\
\hline \multicolumn{5}{|c|}{ IL-23R c.309C>A (rs10889677) } \\
\hline $\mathrm{CC}$ & $88(50.3)$ & $124(59.9)$ & Ref. & \\
\hline $\mathrm{CA}$ & $68(38.9)$ & $69(33.3)$ & $1.39(0.90-2.14)$ & 0.14 \\
\hline AA & $19(10.9)$ & $14(6.8)$ & $1.91(0.91-4.02)$ & 0.09 \\
\hline $\mathrm{CA}+\mathrm{AA}$ & $87(49.7)$ & $83(40.1)$ & $1.48(0.98-2.22)$ & 0.06 \\
\hline HWE & $p=0.29$ & $p=0.31$ & & \\
\hline $\mathrm{C}$ & $244(69.7)$ & $317(76.6)$ & Ref. & \\
\hline A & $106(30.3)$ & $97(23.4)$ & $1.42(1.03-1.96)$ & 0.03 \\
\hline \multicolumn{5}{|c|}{ IL-23R c.1142G>A (rs11209026) } \\
\hline GG & $154(88.0)$ & $179(86.5)$ & Ref. & \\
\hline GA & $20(11.4)$ & $28(13.5)$ & $0.83(0.45-1.53)$ & 0.55 \\
\hline AA & $1(0.6)$ & 0 & $0.29(0.01-7.09)$ & 0.45 \\
\hline $\mathrm{GA}+\mathrm{AA}$ & $21(12.0)$ & $28(13.5)$ & $0.87(0.48-1.60)$ & 0.66 \\
\hline HWE & $p=0.69$ & $p=0.30$ & & \\
\hline G & 328 (93.7) & $386(93.2)$ & Ref. & \\
\hline $\mathrm{A}$ & $22(6.3)$ & $28(6.8)$ & $0.92(0.52-1.65)$ & 0.79 \\
\hline
\end{tabular}


Table 2. Cont.

\begin{tabular}{|c|c|c|c|c|}
\hline Genotype & $\begin{array}{c}\text { Patients } \\
n(\%)\end{array}$ & $\begin{array}{c}\text { Controls } \\
n(\%)\end{array}$ & OR $(95 \% \mathrm{CI})$ & $p$-value \\
\hline \multicolumn{5}{|c|}{ IL-8 -251A >T (rs4073) } \\
\hline AA & $48(27.6)$ & $48(23.2)$ & Ref. & \\
\hline $\mathrm{AT}$ & $75(43.1)$ & $103(49.8)$ & $0.73(0.44-1.20)$ & 0.21 \\
\hline TT & $51(29.3)$ & $56(27.1)$ & $0.91(0.52-1.58)$ & 0.74 \\
\hline $\mathrm{AT}+\mathrm{TT}$ & $126(72.4)$ & $159(76.8)$ & $0.79(0.50-1.26)$ & 0.33 \\
\hline HWE & $p=0.07$ & $p=0.96$ & & \\
\hline A & $171(49.1)$ & $199(48.1)$ & Ref. & \\
\hline $\mathrm{T}$ & $177(50.9)$ & $215(51.9)$ & $1.04(0.78-1.39)$ & 0.77 \\
\hline \multicolumn{5}{|c|}{ IL-2 -330T>G (rs2069762) } \\
\hline TT & $76(43.4)$ & $100(48.3)$ & Ref. & \\
\hline TG & $86(49.1)$ & $92(44.4)$ & $1.23(0.81-1.87)$ & 0.33 \\
\hline GG & $13(7.4)$ & $15(7.2)$ & $1.14(0.51-2.54)$ & 0.75 \\
\hline TG+GG & $99(56.6)$ & $107(51.7)$ & $1.22(0.81-1.82)$ & 0.34 \\
\hline HWE & $p=0.09$ & $p=0.32$ & & \\
\hline $\mathrm{T}$ & $238(68.0)$ & $292(70.5)$ & Ref. & \\
\hline G & $112(32.0)$ & $122(29.5)$ & $0.89(0.65-1.21)$ & 0.45 \\
\hline
\end{tabular}

studied groups $(p=0.12)$. In particular, it was observed that allele A carriers (genotype AA and CA) had about 1.5 fold risk of developing $\mathrm{BC}$, when compared to $\mathrm{CC}$ genotype individuals (OR: $1.48 ; 95 \%$ CI: $0.98-2.22 ; p=0.06$ ).

When IL-17A -197G>A polymorphism was analyzed, statistically different genotypes distribution was also observed $\left(\chi^{2}=6.69, p=0.04\right)$. The heterozygote GA was more frequently found in patients' population (OR: 1.53; 95\% CI: $0.99-2.36 ; p=0.06$ ), as compared to GG individuals. The presence of heterozygosity increased the risk of BC 1.65 fold as compared to both homozygotes (OR: 1.67; 95\% CI: $1.11-2.50 ; p=0.01)$.

Similarly for IL-18 -137G >C polymorphism, heterozygote $\mathrm{GC}$ individuals were more prone to $\mathrm{BC}$ as compared to wild type homozygotes (OR: 1.84 ; $95 \%$ CI: 1.22-2.27; $p=0.001)$ and as compared to both types of homozygotes (OR: 1.84 ; 95\% CI: $1.22-2.79 ; p=0.008$ ).

None of the other polymorphisms were associated with the risk of disease.

\section{Gene-gene interactions and $\mathrm{BC}$ risk}

To analyze the effect of polymorphisms combination, IL-17, IL-18, and IL-23 SNP gene-gene combination analysis was performed. The results are presented in Table 3. The most evident associations were shown for IL-18 -137[GC] and IL-17A -197[GA] genotypes combination with $\mathrm{OR}$ for $\mathrm{BC}$ occurrence risk reaching almost 3 . Additionally, a combination of IL-18 -137[GC] geno- type and IL-23R c.309[CA] or [AA] genotypes as well as combination of IL-17A -197[GA] genotype with IL-23R c.309[CA] genotype were associated with increased BC risk (Table 3). However, after applying Bonferonni correction of multiple comparisons, significance was lost in majority of cases except the combination of IL-18 $-137 \mathrm{G}>\mathrm{C}[\mathrm{GC}]$ and IL-17A $-197 \mathrm{G}>\mathrm{A}[\mathrm{GA}]$ genotypes.

\section{BCG immunotherapy outcome in relation to cytokine gene polymorphisms}

To estimate the association between gene polymorphisms and risk of $\mathrm{BC}$ recurrence and progression additional analysis was performed in BC group $(n=175)$.

The patients with heterozygous GC genotype of IL-18 -137G >C were observed to have the highest risk of recurrence when adjusted for primary diagnosis, age, sex, tumor size, focality, and recurrence status $(\mathrm{HR}=1.79$, $p=0.015)$. Furthermore, Kaplan-Meier analysis for both total NMIBC group and primary diagnosis subgroups was performed. It showed the worst RFS for GC genotype (log rank, $p=0.002$ ), when compared with both homozygotes (Fig. 1A). Similarly, the highest cancer progression risk was associated with heterozygous genotype ( $p=$ $0.013, \mathrm{HR}=2.2$ ) as well as worst progression-free survival (Kaplan-Meier analysis: $\log$ rank, $p=0.022$ ) (Fig. 1B). Results of subgroup analysis were analogous (data not shown).

The IL-17A -197G >A polymorphism was also associated with RFS. Homozygous IL-17A-197G >A[GG] pa- 
Table 3. Gene-gene interactions of IL-17, IL-18, and IL-12 gene polymorphism in BC patients and controls

\begin{tabular}{|c|c|c|c|c|}
\hline & $\begin{array}{c}\text { BC } \\
n(\%)\end{array}$ & $\begin{array}{c}\text { Controls } \\
n(\%)\end{array}$ & OR $(95 \% \mathrm{CI})$ & $p$-value \\
\hline \multicolumn{5}{|c|}{ IL-18 -137G>C (rs187238) and IL-17A -197G>A (rs2275913) } \\
\hline GG-GG & $6(3.4)$ & $10(4.8)$ & & \\
\hline GG-GA & $4(2.3)$ & $10(4.8)$ & $0.46(0.41-0.51)$ & 0.176 \\
\hline GG-AA & $2(1.1)$ & $2(1.0)$ & $1.18(1.16-1.21)$ & 0.865 \\
\hline GC-GG & $26(14.9)$ & $30(14.5)$ & $1.03(1.03-1.03)$ & 0.914 \\
\hline GC-GA & $50(28.6)$ & $25(12.1)$ & $2.91(2.52-3.37)$ & $<0.001$ \\
\hline GC-AA & $6(3.4)$ & $13(6.3)$ & $0.53(0.49-0.58)$ & 0.187 \\
\hline CC-GG & $28(16.0)$ & $47(22.7)$ & $0.65(0.61-0.69)$ & 0.062 \\
\hline CC-GA & $43(24.6)$ & $54(26.1)$ & $0.92(0.91-0.92)$ & 0.693 \\
\hline CC-AA & $9(5.1)$ & $16(7.7)$ & $0.65(0.61-0.69)$ & 0.289 \\
\hline \multicolumn{5}{|c|}{ IL-18 -137G>C (rs187238) and IL-23R c.309C >A (rs10889677) } \\
\hline GG-CC & $3(1.7)$ & $16(7.7)$ & & \\
\hline GG-CA & $7(4.0)$ & $5(2.4)$ & $1.68(1.57-1.81)$ & 0.371 \\
\hline GG-AA & $2(1.1)$ & $1(0.5)$ & $2.38(2.11-2.68)$ & 0.466 \\
\hline $\mathrm{GC}-\mathrm{CC}$ & $40(22.9)$ & $44(21.3)$ & $1.10(1.08-1.11)$ & 0.671 \\
\hline GC-CA & $30(17.1)$ & $18(8.7)$ & $2.17(1.95-2.42)$ & 0.009 \\
\hline GC-AA & $13(7.4))$ & $6(2.9)$ & $2.69(2.35-3.08)$ & 0.039 \\
\hline $\mathrm{CC}-\mathrm{CC}$ & $45(25.7)$ & $64(30.9)$ & $0.77(0.75-0.80)$ & 0.177 \\
\hline $\mathrm{CC}-\mathrm{CA}$ & $31(17.7)$ & $46(22.2)$ & $0.75(0.72-0.78)$ & 0.215 \\
\hline CC-AA & $4(2.3)$ & $7(3.4)$ & $0.67(0.63-0.71)$ & 0.516 \\
\hline \multicolumn{5}{|c|}{ IL-17A -197G >A (rs2275913) and IL-23R c.309C >A (rs10889677) } \\
\hline GG-CC & $31(17.7)$ & $53(25.6)$ & & \\
\hline GG-CA & $23(13.1)$ & $29(14.0)$ & $0.93(0.92-0.94)$ & 0.791 \\
\hline GG-AA & $8(4.6)$ & $5(2.4)$ & $1.94(1.77-2.12)$ & 0.241 \\
\hline GA-CC & $47(26.9)$ & $54(26.1)$ & $1.04(1.03-1.05)$ & 0.843 \\
\hline GA-CA & $39(22.3)$ & $27(13.0)$ & $1.91(1.75-2.09)$ & 0.011 \\
\hline GA-AA & $11(6.3)$ & $8(3.9)$ & $1.67(1.56-1.79)$ & 0.269 \\
\hline AA-CC & $10(5.7)$ & $17(8.2)$ & $0.68(0.64-0.71)$ & 0.322 \\
\hline AA-CA & $6(3.4)$ & $13(6.3)$ & $0.53(0.49-0.58)$ & 0.187 \\
\hline AA-AA & $0(0)$ & $1(0.5)$ & $\mathrm{NC}$ & $\mathrm{NC}$ \\
\hline
\end{tabular}

tients presented the best RFS (log rank, $p=0.045)$ (Fig. 2), while homozygous IL-17A -197G>A[AA] patients were found to have 1.8 fold chance of recurrence development $(\mathrm{HR}=1.81, p=0.031)$. None of the other gene polymorphisms was associated with the recurrence-free and progression-free survival after BCG therapy.

\section{Discussion}

According to international clinical guidelines, intravesical immunotherapy with BCG is the 'gold standard' adjuvant therapy for intermediate- and high-risk NMIBC. However, the risk of HG tumor progression, even with an appropriate surgical and adjuvant treatment, may reach up to $30 \%$ of patients [20]. Additionally, these patients present worse prognosis after radical cystectomy than patients treated with radical cystectomy because of primary MIBC [21]. In spite of the availability of various clinic-pathological prognostic and predictive factors, none of them have proven to be sufficient in every day practice. Moreover, there are no biomarkers to reliable identify candidates for early radical treatment. 
A

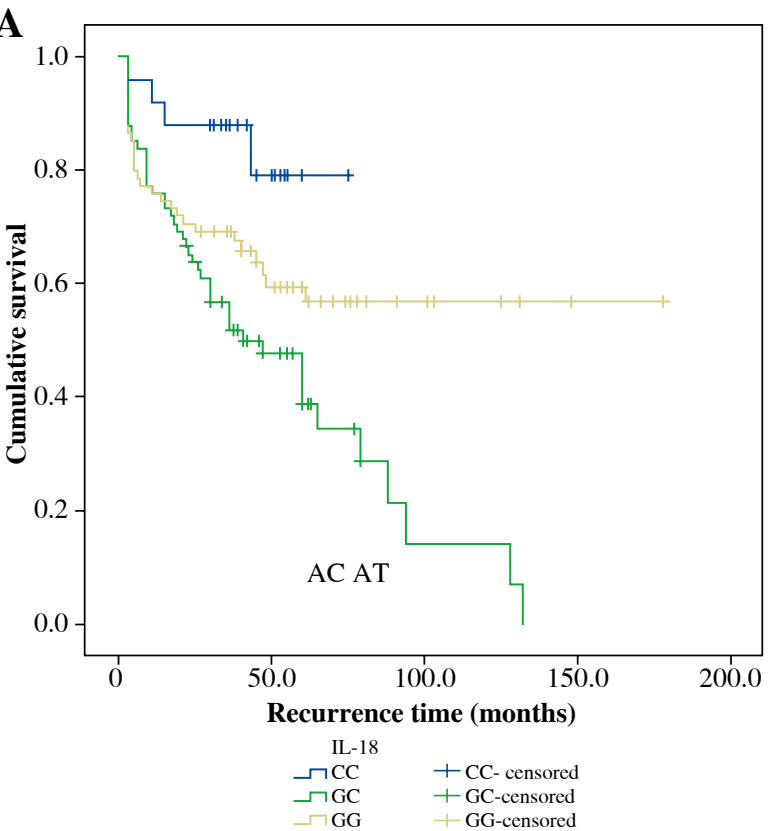

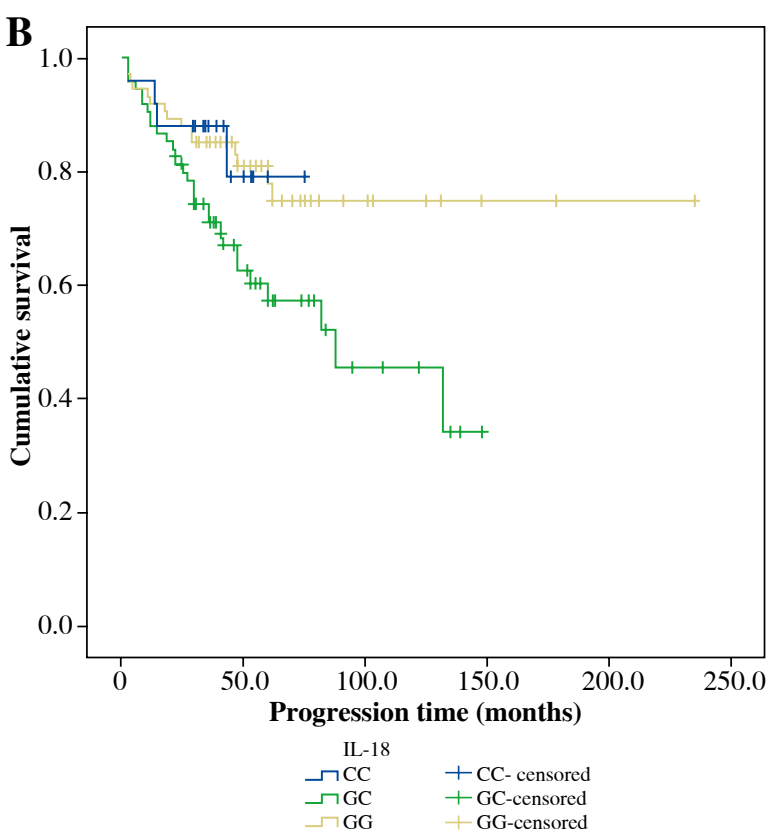

Fig. 1. Kaplan-Meier survival analysis in relation to IL-18 -137G $>C$ (rs187238). A) Recurrence-free survival (log rank, $p=0.002), \mathbf{B})$ progression-free survival (log rank, $\mathrm{p}=0.022$ )

The inflammation is a key element of a urinary bladder malignancy pathogenesis. On the other hand, the mechanism of anti-tumor BCG immunotherapy action is meant to strengthen the immune response by increasing the production of a variety of cytokines.

The potential of immune cells to produce cytokines differs among individuals due to some genetic disparities. Therefore, genetic variations in cytokine genes might be an important determinant of susceptibility to BC and of therapeutic response.

In this study, the association between selected cytokine polymorphisms and BC risk as well as BCG immunotherapy outcome was evaluated in 175 high-risk NMIBC patients.

We found that IL-18 $-137 \mathrm{G}>\mathrm{C}[\mathrm{GC}]$ heterozygosity was associated with higher risk of disease as well as with higher risk of BC recurrence and progression after treatment. Also, patients who presented with combination of heterozygous genotype GC of IL-18 $-137 \mathrm{G}>\mathrm{C}$ and heterozygous genotype GA of IL-17A -197G $>$ A as well as GC genotype of IL-18 $-137 \mathrm{G}>\mathrm{C}$ with CA or AA genotypes of IL-23R c.309C $>\mathrm{A}$ were shown to have increased risk of BC development.

The IL-18, also known as interferon (IFN)- $\gamma$-inducing factor, is a pleiotropic pro-inflammatory cytokine with a crucial role in the Th1 pro-inflammatory response driving. It stimulates IFN- $\gamma$ production in $\mathrm{T}$ and NK cells, induces proliferation of activated $\mathrm{T}$ cells, activation of $\mathrm{NK}$ cells, secretion of numerous chemokines, provokes cancer

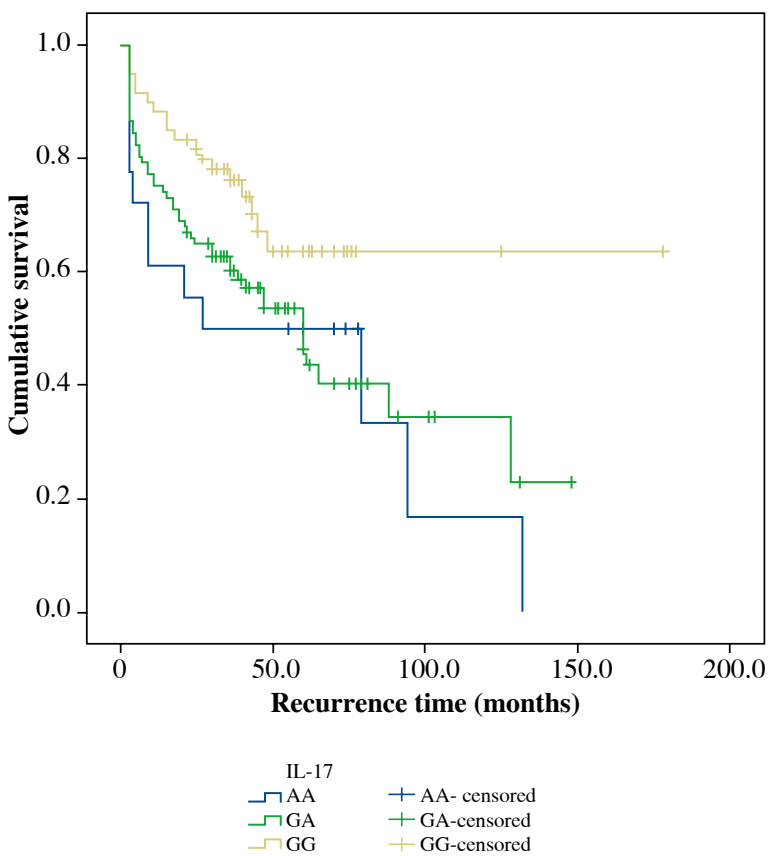

Fig. 2. Kaplan-Meier recurrence-free survival analysis in relation to IL-17A -197G > A (rs2275913) (log rank, $p=0.045$ )

cell apoptosis, and inhibits neoangiogenesis [22, 23]. It is produced by many cells such as dendritic, Kupffer, Langerhans, and activated macrophages [24]. Various SNPs in 
the promoter region of the IL-18 gene seem to modulate gene expression at the transcriptional level. It is postulated that a transition at position -137 from $\mathrm{G}$ to $\mathrm{C}$ at polymorphic site IL-18 -137G $>$ C changed the human histone H4 gene-specific transcription factor-1 nuclear factor binding site [25].

The results of our study are consistent with data obtain by Jaiswal et al. [23]. The authors showed that patients with IL-18 $-137 \mathrm{G}>\mathrm{C}[\mathrm{GC}]$ heterozygous genotype demonstrated two times increased risk of BC. This was also observed by other authors in different malignancies like esophageal squamous cell, lung, prostate, and cervical cancers [26-29]. When NMIBC patients treated with BCG were analyzed in Jaiswal's study, higher risk of recurrence was observed for heterozygous genotype. In the Kaplan-Meier curves analysis, visual trend for lower recurrence-free survival for heterozygous genotype was observed; however, because of short follow-up, statistical significance was not achieved. Authors did not assess progression risk and progression-free survival. Additionally, the authors showed that IL-18 serum levels were significantly higher in BCG patients as compared with healthy individuals. What is more, the highest level of IL-18 was observed in controls BCG patients carrying IL-18$137 \mathrm{G}>\mathrm{C}[\mathrm{GC}]$ heterozygous genotype [23]. Unfortunately, the expression of the IL-18 was not assessed in our study.

The next polymorphisms, which we found associated with disease risk and BCG outcome was IL17A -197G>A. In our study, patients carring A allele IL17A-197G >A [GA +/- AA] genotype had a higher risk of BC development. Also, a combination of IL-17A -197G >A[GA] genotype with IL-23R c.309C $>$ A[CA] genotype was shown to be associated with increased BC risk. Moreover, homozygous GG patients demonstrated the best RFS in survival analysis.

The IL-17 produced by T helper 17 (Th17) subset of CD4 T cells plays an important role in the inflammation process by stimulating release of pro-inflammatory and neutrophil-mobilizing cytokines. Up to date, several IL-17 family ligands and few receptors have been identified. Among the IL-17 family members, IL-17A and IL-17F share the highest amino acid sequence identity and bind to the same receptor. Both IL-17A and IL-17F are involved in the development of inflammation and host defense against infection by inducing the expression of genes encoding pro-inflammatory cytokines (TNF, IL-1, IL-6, G-CSF, and GM-CSF), chemokines (CXCL1, CXCL5, IL-8, CCL2, and CCL7), antimicrobial peptides (defensins and $\mathrm{S} 100$ proteins), and matrix metalloproteinases (MMP1, MMP3, and MMP13) from fibroblasts, endothelial cells, and epithelial cells [30, 31]. It was shown that IL-17 may play a key role in the recruitment of neutrophils to the bladder after BCG immunotherapy, which is essential for the anti-cancer activity [32]. The literature data related to IL-17A - $197 \mathrm{G}>$ A polymorphism are consistent with our observations. In the study conducted by Zhou et al. it was shown that allele A of IL-17A -197G >A was associated with the development of BC [30]. In other study performed on European population it was indicated that patients carrying the IL-17A -197G >A[AA] genotype presented an increased risk of both $\mathrm{BC}$ occurrence and recurrence after BCG treatment [33]. In Li's paper, the presence of AA genotype of IL-17A -197G>A polymorphism has been linked to diminished levels of this interleukin expression and as postulated by the authors, it might cause decreased response to treatment [34].

The next polymorphism occurring in IL-17 family, IL-17F +7488T >C was described as associated with susceptibility to BC in Zhou et al. publication; however our study performed on Caucasian population did not confirm their results [30].

We also found that A allele in IL-23R c.309C >A SNP was related to susceptibility to BC. Moreover, the borderline association between presence of A allele (CA and AA genotype) and risk of $\mathrm{BC}$ was observed. Interleukin-23 (IL-23) and IL-23 receptor (IL-23R) play an important role during the T-helper 17 (Th17) cell-mediated inflammatory process as well as pathogenesis of multiple cancers. The IL-23/IL-23R interaction promotes pro-inflammatory processes engaged in tumor development and is responsible for the failure of adaptive immune surveillance [35]. It was reported that IL-23R could also decrease immunosurveillance by CD8+ T cells and promote tumor growth [36]. In tumor microenvironment, IL-23R signaling pathway also promote the immunosuppressive function of $\mathrm{T}$ regulatory cell (Tregs), what additionally enables evasion of the immune system by cancer cells [37, 38].

The various polymorphisms in gene encoding IL-23R were found to be a susceptibility locus for many cancers [39-44]. In particular, IL-23R c.309C>A [AA] genotype was linked to increased risk of cancer development while possessing of $\mathrm{C}$ allele to decreased risk of the disease [45]. This polymorphism is located in the binding sequence of let-7F microRNA precursor and is the factor, which could negatively regulate IL-23R expression [45, 46]. It was shown that healthy individuals possessing AA genotype of IL-23R c.309C > A polymorphism presented higher expression of IL-23R in peripheral blood mononuclear cells, when compared to the individuals possessing $C$ allele [46]. That data suggested that presence of A allele is associated with higher expression of IL-23R and as a consequence promotes tumor development.

On the other hand, in the paper by Tang et al., the authors found a significant association between IL-23R c.309C $>$ A SNP and BC risk, while the distribution of genotypes was different from observed in Polish population. According to population data, there is an inverse allele distribution for this particular SNP between European (C-70\% and A-30\%) and South Asian population (C-40\% and A-60\%), what might explain the observed discrepancies [47]. 
For IL-23R c.1142G>A polymorphism, no significant differences for the allele and genotype distributions between patients and controls were observed. Correspondingly, no influence on RFS nor PFS was found for this SNP.

We have also investigated IL-2 $-330 \mathrm{~T}>\mathrm{G}$ polymorphism. It lies in the promoter region of the IL-2 gene, and was proved to be implicated in the susceptibility to a range of inflammation diseases and cancers [48]. The IL-2 is secreted by activated T CD4+ cells and plays a central role in the activation of cytotoxic $\mathrm{T}$, functioning as a T cell growth factor. Also, IL-2 contributes to the development of regulatory $\mathrm{T}$ cells and controls the expansion and apoptosis among activated $\mathrm{T}$ cells, and therefore plays the pivotal role in antitumor immunity [49]. In the study conducted by Shen et al. it was shown that frequencies of the GG IL-2 -330T>G genotype as well as G allele were significantly higher in BC patients than in controls [48]. In our population, none of above mentioned results were confirmed. Neither the IL-2 -330T > G genotypes, nor the alleles were associated with $\mathrm{BC}$ susceptibility or response to BCG treatment. It may be caused by the difference in patients' ethnicity.

The last polymorphisms selected for this study was IL-8 -251A $>$ T. The IL-8 is a pro-inflammatory cytokine associated with the promotion of neutrophil chemotaxis and degranulation. Increased expression of IL- 8 and/or its receptors has been characterized in cancer cells (including bladder cancer), endothelial cells, infiltrating neutrophils, and tumor-associated macrophages, suggesting that IL-8 may function as a significant regulatory factor within the tumor microenvironment [50, 51]. Additionally, as it was previously noted, IL-8 secretion after the initial intravesical BCG instillation strongly correlates with the treatment outcome [52]. These observations suggest that IL- 8 genes may play a role in BC susceptibility and therapy outcome. In the study on Indian population it was found that both patients with homozygote AA and A allele carriers presented an increased risk of $\mathrm{BC}$ development. It was also shown that AA genotype was associated with better RFS [52]. On the other hand, in the study conducted by Leibovici et al. on Caucasian population, the authors did not find significant associations between variant genotypes for IL-8 and BC risk, neither for treatment outcome [53]. Similarly to Leibovici, we did not find any associations of this polymorphism with $\mathrm{BC}$ and response to BCG therapy.

\section{Limitations and strengths}

Firstly, this study was limited by its retrospective nature. There was a possible selection bias that may have been present in the hospital-based, case-control study. Additionally, the long period of the observation raises doubts about whether a cohort effect may occur. However, during more than 20 years of the BCG outpatient clinic functioning, none of qualification, therapeutic, and maintenance details changed. Furthermore, to avoid biases, only one team of physicians qualified all patients and one physician administered almost all of instillations (AK). Additionally, only two nurses were included in the treatment protocols.

Secondly, we did not assess the levels of genes expressions. However, those molecules can only be reliably measured during tumor development and anti-cancerous treatment periods, and therefore analysis performed years later would not be dependable.

Thirdly, we did not analyze smoking status impact. However, this risk factor has been previously widely proven to be associated with $\mathrm{BC}$ susceptibility and worse treatment results.

Despite limitations, our study has some clear strengths. Firstly, we included the most clinically important and homogenous group of cancers. Is is well known that compering to other NMIBC subgroups, high-grade cancers harbor the highest risk of progression and cancer-specific death. Secondly, in contrary to other studies, our relatively small group was very homogenous in regard with stage, grade, and treatment protocol, and was followed-up meticulously for a long period of time. Finally, available publications focus mostly on North Indian and Chinese population. We present study on European population, which has been rarely addressed before.

\section{Conclusions}

The present study has highlighted the importance of IL-17, IL-18, and IL-23R gene polymorphisms and bladder cancer susceptibility. Additionally, the study has proven that IL-17 and IL-18 genes polymorphisms might be associated with BCG immunotherapy outcome. Those findings may improve personalized clinical decision making and identify patients, which are best candidates for early radical treatment.

\section{Acknowledgments}

The study was supported by institutional Wroclaw Medical University scientific grant entitled "Cytokine gene polymorphism as predisposing, prognostic and predictive of BCG immunotherapy response in bladder cancer" (Pbm194).

The authors declare no conflict of interest.

\section{References}

1. Siegel RL, Miller KD, Jemal A (2017): Cancer Statistics, 2017. CA Cancer J Clin 67: 7-30.

2. Babjuk M, Bohle A, Burger M, et al. (2017): EAU Guidelines on Non-Muscle-invasive Urothelial Carcinoma of the Bladder: Update 2016. Eur Urol 71: 447-461.

3. Balkwill F, Mantovani A (2001): Inflammation and cancer: back to Virchow? Lancet 357: 539-545. 
4. Groah SL, Weitzenkamp DA, Lammertse DP, et al. (2002): Excess risk of bladder cancer in spinal cord injury: evidence for an association between indwelling catheter use and bladder cancer. Arch Phys Med Rehabil 83: 346-351.

5. Redelman-Sidi G, Glickman MS, Bochner BH (2014): The mechanism of action of BCG therapy for bladder cancer a current perspective. Nat Rev Urol 11: 153-162.

6. Alexandroff AB, Nicholson S, Patel PM, et al. (2010): Recent advances in bacillus Calmette-Guerin immunotherapy in bladder cancer. Immunotherapy 2: 551-560.

7. Gu J, Grossman HB, Dinney CP, et al. (2005): The pharmacogenetic impact of inflammatory genes on bladder cancer recurrence. Pharmacogenomics 6: 575-584.

8. Lima L, Dinis-Ribeiro M, Longatto-Filho A, et al. (2012): Predictive biomarkers of bacillus calmette-guerin immunotherapy response in bladder cancer: where are we now? Adv Urol 2012: 232609.

9. Ahirwar D, Kesarwani P, Manchanda PK, et al. (2008): Antiand proinflammatory cytokine gene polymorphism and genetic predisposition: association with smoking, tumor stage and grade, and bacillus Calmette-Guerin immunotherapy in bladder cancer. Cancer Genet Cytogenet 184: 1-8.

10. Sobin LH, Gospodarowicz MK (2010): TNM classification of malignant tumours. Wittekind CInternational Union Against Cancer. $7^{\text {th }}$ ed. Wiley-Blackwell, New York.

11. Lamm DL, Blumenstein BA, Crissman JD, et al. (2000): Maintenance bacillus Calmette-Guerin immunotherapy for recurrent TA, T1 and carcinoma in situ transitional cell carcinoma of the bladder: a randomized Southwest Oncology Group Study. J Urol 163: 1124-1129.

12. Matesanz F, Fedetz M, Collado-Romero M, et al. (2001): Allelic expression and interleukin-2 polymorphisms in multiple sclerosis. J Neuroimmunol 119: 101-105.

13. Hull J, Thomson A, Kwiatkowski D (2000): Association of respiratory syncytial virus bronchiolitis with the interleukin 8 gene region in UK families. Thorax 55: 1023-1027.

14. Pravica V, Perrey C, Stevens A, et al. (2000): A single nucleotide polymorphism in the first intron of the human IFN-gamma gene: absolute correlation with a polymorphic CA microsatellite marker of high IFN-gamma production. Hum Immunol 61: 863-866.

15. Espinoza JL, Takami A, Nakata K, et al. (2011): A genetic variant in the IL-17 promoter is functionally associated with acute graft-versus-host disease after unrelated bone marrow transplantation. PLoS One 6: e26229.

16. Kawaguchi M, Takahashi D, Hizawa N, et al. (2006): IL-17F sequence variant (His161 Arg) is associated with protection against asthma and antagonizes wild-type IL-17F activity. J Allergy Clin Immunol 117: 795-801.

17. Tiret L, Godefroy T, Lubos E, et al. (2005): Genetic analysis of the interleukin-18 system highlights the role of the interleukin-18 gene in cardiovascular disease. Circulation 112: 643-650.

18. Xu Z, Taylor JA (2009): SNPinfo: integrating GWAS and candidate gene information into functional SNP selection for genetic association studies. Nucleic Acids Res 37: W600-605.

19. Lee WC (2003): Searching for disease-susceptibility loci by testing for Hardy-Weinberg disequilibrium in a gene bank of affected individuals. Am J Epidemiol 158: 397-400.

20. Gontero P, Sylvester R, Pisano F, et al. (2015): Prognostic factors and risk groups in T1G3 non-muscle-invasive bladder cancer patients initially treated with Bacillus Calmette-Guer- in: results of a retrospective multicenter study of 2451 patients. Eur Urol 67: 74-82.

21. van den Bosch S, Alfred Witjes J (2011): Long-term cancer-specific survival in patients with high-risk, non-muscle-invasive bladder cancer and tumour progression: a systematic review. Eur Urol 60: 493-500.

22. Nakanishi K, Yoshimoto T, Tsutsui H, et al. (2001): Interleukin-18 is a unique cytokine that stimulates both Th1 and Th2 responses depending on its cytokine milieu. Cytokine Growth Factor Rev 12: 53-72.

23. Jaiswal PK, Singh V, Srivastava P, et al. (2013): Association of IL-12, IL-18 variants and serum IL-18 with bladder cancer susceptibility in North Indian population. Gene 519: 128-134.

24. Baxevanis CN, Gritzapis AD, Papamichail M (2003): In vivo antitumor activity of NKT cells activated by the combination of IL-12 and IL-18. J Immunol 171: 2953-2959.

25. Giedraitis V, He B, Huang WX, et al. (2001): Cloning and mutation analysis of the human IL-18 promoter: a possible role of polymorphisms in expression regulation. J Neuroimmunol 112: 146-152.

26. Wei YS, Lan Y, Liu YG, et al. (2007): Interleukin-18 gene promoter polymorphisms and the risk of esophageal squamous cell carcinoma. Acta Oncol 46: 1090-1096.

27. Farjadfar A, Mojtahedi Z, Ghayumi MA, et al. (2009): Interleukin-18 promoter polymorphism is associated with lung cancer: a case-control study. Acta Oncol 48: 971-976.

28. Liu Y, Lin N, Huang L, et al. (2007): Genetic polymorphisms of the interleukin-18 gene and risk of prostate cancer. DNA Cell Biol 26: 613-618.

29. Sobti RC, Shekari M, Tamandani DM, et al. (2008): Association of interleukin-18 gene promoter polymorphism on the risk of cervix carcinogenesis in north Indian population. Oncol Res 17: 159-166.

30. Zhou B, Zhang P, Wang Y, et al. (2013): Interleukin-17 gene polymorphisms are associated with bladder cancer in a Chinese Han population. Mol Carcinog 52: 871-878.

31. Iwakura Y, Ishigame H, Saijo S, et al. (2011): Functional specialization of interleukin-17 family members. Immunity 34: 149-162.

32. Takeuchi A, Dejima T, Yamada H, et al. (2011): IL-17 production by gammadelta $\mathrm{T}$ cells is important for the antitumor effect of Mycobacterium bovis bacillus Calmette-Guerin treatment against bladder cancer. Eur J Immunol 41: 246-251.

33. Lima L, Oliveira D, Ferreira JA, et al. (2015): The role of functional polymorphisms in immune response genes as biomarkers of bacille Calmette-Guerin (BCG) immunotherapy outcome in bladder cancer: establishment of a predictive profile in a Southern Europe population. BJU Int 116: 753-763.

34. Li N, Zhu Q, Li Z, et al. (2014): IL17A gene polymorphisms, serum IL-17A and IgE levels, and hepatocellular carcinoma risk in patients with chronic hepatitis B virus infection. Mol Carcinog 53: 447-457.

35. Volpe E, Servant N, Zollinger R, et al. (2008): A critical function for transforming growth factor-beta, interleukin 23 and proinflammatory cytokines in driving and modulating human $\mathrm{T}(\mathrm{H})-17$ responses. Nat Immunol 9: 650-657.

36. Langowski JL, Zhang X, Wu L, et al. (2006): IL-23 promotes tumour incidence and growth. Nature 442: 461-465.

37. Fontenot JD, Gavin MA, Rudensky AY (2003): Foxp3 programs the development and function of $\mathrm{CD} 4+\mathrm{CD} 25+$ regulatory T cells. Nat Immunol 4: 330-336. 
38. Hori S, Takahashi T, Sakaguchi S (2003): Control of autoimmunity by naturally arising regulatory $\mathrm{CD} 4+\mathrm{T}$ cells. Adv Immunol 81: 331-371.

39. Chen J, Lu Y, Zhang H, et al. (2010): A nonsynonymous polymorphism in IL23R gene is associated with risk of gastric cancer in a Chinese population. Mol Carcinog 49: 862-868.

40. Chien MH, Hsin CH, Chou LS, et al. (2012): Interleukin-23 receptor polymorphism as a risk factor for oral cancer susceptibility. Head Neck 34: 551-556.

41. Chu H, Cao W, Chen W, et al. (2012): Potentially functional polymorphisms in IL-23 receptor and risk of esophageal cancer in a Chinese population. Int J Cancer 130: 1093-1097.

42. Poole EM, Curtin K, Hsu L, et al. (2012): Genetic variability in IL23R and risk of colorectal adenoma and colorectal cancer. Cancer Epidemiol 36: e104-110.

43. Qian X, Cao S, Yang G, et al. (2013): Potentially functional polymorphism in IL-23 receptor and risk of acute myeloid leukemia in a Chinese population. PLoS One 8: e55473.

44. Xu Y, Liu Y, Pan S, et al. (2013): IL-23R polymorphisms, HBV infection, and risk of hepatocellular carcinoma in a high-risk Chinese population. J Gastroenterol 48: 125-131.

45. Yao J, Liu L, Yang M (2014): Interleukin-23 receptor genetic variants contribute to susceptibility of multiple cancers. Gene 533: 21-25.

46. Zheng J, Jiang L, Zhang L, et al. (2012): Functional genetic variations in the IL-23 receptor gene are associated with risk of breast, lung and nasopharyngeal cancer in Chinese populations. Carcinogenesis 33: 2409-2416.

47. Ensembl genome database. https://www.ensembl.org/index. html

48. Shen Y, Liu Y, Liu S, et al. (2012): The association between $-330 \mathrm{~T} / \mathrm{G}$ polymorphism of interleukin 2 gene and bladder cancer. DNA Cell Biol 31: 983-987.

49. Zhang Y, Luo CL, He BC, et al. (2010): Exosomes derived from IL-12-anchored renal cancer cells increase induction of specific antitumor response in vitro: a novel vaccine for renal cell carcinoma. Int J Oncol 36: 133-140.

50. Black PC, Dinney CP (2007): Bladder cancer angiogenesis and metastasis--translation from murine model to clinical trial. Cancer Metastasis Rev 26: 623-634.

51. Waugh DJ, Wilson C (2008): The interleukin-8 pathway in cancer. Clin Cancer Res 14: 6735-6741.

52. Ahirwar DK, Mandhani A, Mittal RD (2010): IL-8 -251 T > A polymorphism is associated with bladder cancer susceptibility and outcome after BCG immunotherapy in a northern Indian cohort. Arch Med Res 41: 97-103.

53. Leibovici D, Grossman HB, Dinney CP, et al. (2005): Polymorphisms in inflammation genes and bladder cancer: from initiation to recurrence, progression, and survival. J Clin Oncol 23: 5746-5756. 